\title{
Implications of hybrid vigour, combining ability and per se performance in pigeonpea [Cajanus cajan (L.) Millsp.]
}

\author{
Yamanura $^{1 *}$, S. Muniswamy ${ }^{1}$ and Ramesh $^{2}$ \\ ${ }^{1}$ Department of Genetics and Plant Breeding, Univesity of Agricultural Sciences, Raichur-584 102 (Karnataka), INDIA \\ ${ }^{2}$ Agricultural Research Station, Kalaburagi-585101 (Karnataka), INDIA \\ *Corresponding author. E-mail: yaman3181aug8@gmail.com \\ Received: June 15, 2016; Revised received: January 18, 2016; Accepted: April 11, 2016
}

\begin{abstract}
Three CGMS lines were crossed with 17 testers in a line $\times$ tester design during Kharif 2013 and sufficient number of hand pollinated seeds was produced. The resultant 51 hybrids along with their 20 parents and standard check variety (Maruti) were evaluated in RBD design with two replications. Combining ability analysis evinced predominance of non-additive gene effects for 7 characters indicating relevance of heterosis breeding for improving yield attributes. The gca effects of parents revealed that ICPA-2043, GRG-2009, GRG-2009-2, LAXMI, LRG-41 and JKM-197 were good general combiners for seed yield and it's direct components. The estimates of sca effects revealed that 11 experimental hybrids had significant, desirable and positive sca effects for seed yield/plant. Among these, three best crosses were selected on the basis of per se performance for ascertaining their association with sca effects of seed yield per plant and its attributes. The investigation identified the good general combiners (ICPA -2043, GRG-2009, GRG-2009-2, LAXMI, LRG-41 and JKM-197) and promising crosses (ICPA-2043 X GRG-2009-2, ICPA-2047 X GRG-2009 and ICPA-2043 X ICPL-288) showing high mean and significant positive sca effects involved high $\times$ high gca effects of parents. These parental combinations may be used in breeding program for exploitation of hybrid vigour.
\end{abstract}

Keywords: Combining ability, Hybrid vigour, LineX Testers, per se, Pigeonpea

\section{INTRODUCTION}

Pigeonpea (Cajanus cajan (L.) Millsp.) is a perennial shrub belong to economically important tribe Phaseoleae and the subtribe Cajanine. It is being cultivated as an annual crop in Southern and South Eastern Asia, Eastern Africa, the Caribbean region and South and Central America. It is chiefly grown for its seeds which are consumed either as dry split peas (dhal), providing major source of protein and essential amino acids or as a green vegetable (Saxena et al. 2005).

In India, it is one of the very important grain legumes and occupies second position in area and production next to chickpea. It is grown in an area of $3.88 \mathrm{~m}$ ha with an annual production of 3.17 m tonnes. In Karnataka, pigeonpea occupies second place in area $(6.66$ Lakh ha) and ranks second in production (3.66 Lakh tonnes) with a productivity of $555 \mathrm{~kg} / \mathrm{ha}$ (Sing, 2015).

Most of the economic characters like seed yield, number of pods per plant, days to 50 per cent flowering are mostly governed by polygenes and their inheritance is of complex nature. Therefore, before making attempts for improvement of these characters it is essential to know the nature of gene action controlling these quantitative characters. This information will be helpful to breeders in devising appropriate methods of breeding for crop improvement. A review of literature on quan- titative genetics of pigeonpea showed that the presence of significant levels of non additive genetic variation for seed yield which could be profitably exploited through heterosis breeding to increase grain yield (Saxena and Sharma, 1990).

Commercial exploitation of heterosis has been possible in crops like sorghum and cotton either through male sterility systems or through hand pollination. A successful search for easily identifiable and stable genetic male sterility at different institutions in India has paved way for commercial exploitation of hybrid vigour in pigeonpea and six hybrids viz., ICPH 8, COPH 1, COPH 2, PPH 4, AKPH 4104 and AKPH 2022 have been released for commercial cultivation. ( Bajpai et al., 2003)

Exploitation of heterosis depends much on general and specific combining ability effects. Combining ability studies are useful in evaluation of the parental lines and their cross combinations, usually this information aids in selection of parents in terms of performance of hybrids and elucidate the nature and magnitude of various types of gene action involved in the expression of quantitative traits (Sony, 2010). Therefore, present study was undertaken to estimate hybrid vigor, combining ability for seed yield and other traits in pigeonpea using three cytoplasmic genic male sterile lines derived from $\mathrm{A}_{4}$ cytoplasm and 17 diverse testers 
Table 1. Variance due to GCA, SCA ad their proportion for 7 different characters in pigeonpea.

\begin{tabular}{llccc}
\hline S. N. & Characters & Variance due to GCA & Variance due to SCA & GCA : SCA proportion \\
\hline 1 & Days to 50 per cent flowering & 1.18 & 15.55 & $1: 13.13$ \\
2 & Days to maturity & 1.31 & 5.50 & $1: 4.19$ \\
3 & Plant height (cm) & 8.30 & 92.85 & $1: 11.19$ \\
4 & Number of branches/plant & 0.17 & 1.19 & $1: 6.88$ \\
5 & Pod bearing length (cm) & 3.03 & 89.34 & $1: 29.47$ \\
6 & Number of pods/plant & 2.58 & 1269.06 & $1: 492.46$ \\
7 & Yield per plant(gm) & 3.60 & 312.88 & $1: 86.96$ \\
\hline
\end{tabular}

crossed in line $\mathrm{x}$ tester design in pigeonpea.

\section{MATERIALS AND METHODS}

The parental material comprised of three CGMS lines (ICPA-2043, ICPA-2078 and ICPA-2047) used as a females were crossed with seventeen genotypes viz. ICPL-288, ICPL-89004, ICP-6974, ICP-7387, ICP6972, LRG-41, MARUTI, WRP-1, GRG-2009, JKM197, GRG-295, TGT-501, PG-12, GRG-2009-2, GRG2009-3, TS-3 and LAXMI used as a males in line $\mathrm{x}$ tester mating design during Kharif 2013 and sufficient number of hand pollinated seeds were produced. The resultant 51 hybrids along with their 20 parents and standard check variety (Maruti) were evaluated in a RBD design with two replications. Each genotype was sown in two rows of 4.0 meter length with the spacing of $90 \mathrm{~cm}$ between rows and $30 \mathrm{~cm}$ between plants at Agriculture Research Station, Gulbarga during kharif 2014.

Observations on five randomly selected competitive plants were recorded for days to $50 \%$ flowering, days to maturity, plant height $(\mathrm{cm})$, number of primary \& secondary branches/plant, pod bearing length $(\mathrm{cm})$, number of pods/plant, seed yield/plant (g). Fertile male sibs were used to collect data on male sterile lines. The mean value of five random plants of the abovementioned characters recorded on parents and hybrids were utilized and variance due to different sources was estimated (Panse and Sukhatme, 1961).

The variance due to general combining ability (GCA) of parents and specific combining ability (SCA) of different cross combinations were worked out using $\mathrm{L}$ $\mathrm{x} \mathrm{T}$ analysis based on the procedure developed by Kempthorne (1957). The GCA variance was more than the SCA variance i.e. GCA/SCA variance ratio was more than one indicating predominance of additive variance. The SCA variance was higher than the GCA variance i.e. $\mathrm{GCA} / \mathrm{SCA}$ variance ratio was less than unity, indicating predominance of dominance variance than additive variance. The data was subjected to analysis of variance and combining ability using statistic package WINDOSTAT 8.5 developed by Indostat services, Hyderabad (India).

\section{RESULTS AND DISCUSSION}

The results revealed higher magnitude of SCA variance as compared to GCA variance for all the characters indicating the predominance of non-additive gene action. The ratio between GCA and SCA variances were worked out and the proportion was highest for total number of pods per plant $(1: 492.46)$ followed by seed yield per plant $(1: 86.96)$, pod bearing length (1:29.47), day to 50 per cent flowering $(1: 13.13)$, plant height (1:11.19), Number of branches/plant (1:6.88) and days to maturity (1:4.19) and these characters are more likely to be improved through heterosis breeding (Table 1). The above findings are in agreement with the earlier reports of Sunil Kumar et al. (2003), Sekhar et al. (2004), Sameer Kumar et al. (2009), Beekham and Umaharan (2010), Shobha and Balan (2010), Sony Tiwari (2010), Chethana et al. (2013) and Meshram et al. (2013). They reported the same results for most of

Table 2. ANOVA for combining ability in respect of 7 characters in pigeonpea.

\begin{tabular}{lcccccccc}
\hline $\begin{array}{l}\text { Source of } \\
\text { Variation }\end{array}$ & $\begin{array}{c}\text { Degree of } \\
\text { freedom }\end{array}$ & $\begin{array}{c}\text { Days to 50\% } \\
\text { flowering }\end{array}$ & $\begin{array}{c}\text { Days to } \\
\text { maturity }\end{array}$ & $\begin{array}{c}\text { Plant } \\
\text { height } \\
\text { (cm) }\end{array}$ & $\begin{array}{c}\text { Number of } \\
\text { branches } \\
\text { per plant }\end{array}$ & $\begin{array}{c}\text { Pod bear- } \\
\text { ing length } \\
\text { (cm) }\end{array}$ & $\begin{array}{c}\text { No. of } \\
\text { pods/ plant }\end{array}$ & $\begin{array}{c}\text { Seed yield/ } \\
\text { plant }\end{array}$ \\
\hline $\begin{array}{l}\text { Females } \\
\text { Males }\end{array}$ & 2 & $791.18^{* *}$ & $544.89 * *$ & $7105.90 * *$ & $204.39 * *$ & $4527.85 * *$ & 8591.78 & $4149.21 * *$ \\
Females & 16 & $285.94 *$ & $219.15 * *$ & $1142.43 * *$ & 17.86 & 288.20 & 2667.30 \\
x Males & 32 & 147.92 & 39.95 & $443.83 * *$ & 10.13 & $261.96 * *$ & $2902.90 * *$ \\
Error & 50 & 116.81 & 28.95 & 258.67 & 7.75 & 83.28 & 364.77 \\
\hline
\end{tabular}

$*$,* - Significant at $5 \%$ and $1 \%$ levels, respectively

Table 3. Per cent contribution of Lines, Testers and Line X Testers to crosses in pigeonpea.

\begin{tabular}{lccccccc}
\hline $\begin{array}{l}\text { Source of } \\
\text { Variation }\end{array}$ & $\begin{array}{c}\text { Days to 50\% } \\
\text { flowering }\end{array}$ & $\begin{array}{c}\text { Days to } \\
\text { maturity }\end{array}$ & $\begin{array}{c}\text { Plant } \\
\text { height } \mathbf{( c m )}\end{array}$ & $\begin{array}{c}\text { Number of } \\
\text { branches per plant }\end{array}$ & $\begin{array}{c}\text { Pod bearing } \\
\text { length (cm) }\end{array}$ & $\begin{array}{c}\text { No. of } \\
\text { pods/ plant }\end{array}$ & $\begin{array}{c}\text { Seed yield/ } \\
\text { plant }\end{array}$ \\
\hline Lines & 14.53 & 18.55 & 30.44 & 40.13 & 41.07 & 11.25 \\
Testers & 42.01 & 59.69 & 39.15 & 28.04 & 20.91 & 27.94 \\
LinesX & 43.46 & 21.76 & 30.42 & 31.83 & 38.02 & 60.81 \\
Testers & & & & & 48.31 & \\
\hline
\end{tabular}


Table 4. General combining ability effects for parents in respect of 7 characters in pigeopea.

\begin{tabular}{|c|c|c|c|c|c|c|c|}
\hline Entries & $\begin{array}{c}\text { Days to } 50 \% \\
\text { flowering }\end{array}$ & $\begin{array}{l}\text { Days to } \\
\text { maturity }\end{array}$ & $\begin{array}{c}\text { Plant } \\
\text { height }(\mathrm{cm})\end{array}$ & $\begin{array}{c}\text { Number of } \\
\text { branches } \\
\text { per plant }\end{array}$ & $\begin{array}{l}\text { Pod bearing } \\
\text { length }(\mathrm{cm})\end{array}$ & $\begin{array}{l}\text { No. of } \\
\text { pods/ } \\
\text { plant }\end{array}$ & $\begin{array}{c}\text { Seed yield/ } \\
\text { plant }\end{array}$ \\
\hline \multicolumn{8}{|l|}{ LINES } \\
\hline ICPA-2043 & -0.75 & -1.25 & -4.14 & $2.57 * *$ & 1.51 & $18.25 * *$ & $10.45 * *$ \\
\hline ICPA2078 & $-4.40 *$ & $-3.23 * *$ & $-11.93 * *$ & $-2.31 * *$ & $-12.22 * *$ & $-7.46 *$ & $-11.56 * *$ \\
\hline ICPA-2047 & $5.16^{* *}$ & $4.48 * *$ & $16.08 * *$ & -0.27 & $10.71 * *$ & $-10.79 * *$ & 1.11 \\
\hline $\mathrm{CD} @ 5 \%$ & 5.26 & 2.62 & 7.84 & 1.35 & 4.44 & 9.31 & 2.59 \\
\hline CD@1\% & 7.02 & 3.49 & 10.45 & 1.8 & 5.93 & 12.41 & 3.45 \\
\hline \multicolumn{8}{|l|}{ TESTERS } \\
\hline ICPL-288 & -3.96 & $-4.87 *$ & -10.85 & -0.22 & 5.1 & $30.62 * *$ & $9.71 * *$ \\
\hline ICPL-89004 & -6.29 & $-7.21 * *$ & -8.35 & -1.56 & 1.54 & 9.68 & -1.77 \\
\hline ICP-6974 & 0.71 & -0.21 & -9.91 & 0.61 & -2.79 & 14.9 & $-11.35 * *$ \\
\hline ICP-7387 & 3.04 & 2.13 & -1.13 & 1.17 & -2.62 & -15.21 & $-8.54 * *$ \\
\hline ICP-6972 & $-11.63 *$ & $-12.54 * *$ & $-28.13 * *$ & $-3.83 * *$ & $-9.74 *$ & $-34.60 * *$ & $-23.33 * *$ \\
\hline LRG-41 & $-12.96 * *$ & 4.29 & 5.54 & 0.94 & 0.82 & $16.02 *$ & $8.95 * *$ \\
\hline MARUTI & 1.37 & 2.29 & 10.04 & -0.11 & $10.65 * *$ & $-15.82 *$ & $-8.95 * *$ \\
\hline WRP-1 & 4.21 & 3.29 & 11.31 & -1.95 & $17.26 * *$ & $-17.49 *$ & $-13.60 * *$ \\
\hline GRG-2009 & 3.21 & 2.29 & 4.98 & 0.67 & -5.51 & $-20.54 *$ & $18.60 * *$ \\
\hline JKM-197 & $15.21 * *$ & $13.13 * *$ & $15.37 *$ & 1.55 & 4.1 & $44.35 * *$ & $4.51 *$ \\
\hline GRG-295 & 3.21 & 2.29 & 3.98 & $3.55 * *$ & -3.68 & 9.01 & 3.4 \\
\hline TGT-501 & -0.46 & -1.37 & 11.03 & 0.94 & $-9.35 *$ & 7.46 & -3.71 \\
\hline PG-12 & -5.79 & $-6.71 * *$ & $-31.96 * *$ & -1.87 & -3.07 & $-16.93 *$ & $-6.51 * *$ \\
\hline GRG-2009-2 (209) & -0.46 & -2.54 & 1.87 & -0.17 & -0.51 & 7.73 & $20.11 * *$ \\
\hline GRG-2009-3 (216) & 1.04 & -2.04 & 2.65 & -0.95 & 2.54 & -7.27 & $-7.47 * *$ \\
\hline TS-3 & $9.37 *$ & $8.46 * *$ & 11.92 & -0.5 & -7.01 & $-23.60 * *$ & 4.17 \\
\hline LAXMI & 0.21 & -0.71 & 11.65 & 1.72 & 2.26 & 11.68 & $15.79 * *$ \\
\hline CD@5\% & 12.54 & 6.24 & 18.66 & 3.23 & 10.59 & 22.16 & 6.17 \\
\hline CD@1\% & 16.72 & 8.32 & 24.88 & 4.3 & 14.12 & 29.55 & 8.23 \\
\hline
\end{tabular}

*, ** - Significant at $5 \%$ and $1 \%$ levels, respectively

the important characters including seed yield except plant height indicating these traits are under the influence of non additive gene action.

Analysis of variance for combining ability revealed that mean squares due to females were significant for all most all the characters except number of pods per plant while mean squares due to males were significant for characters viz., days to $50 \%$ flowering, days to maturity and plant height $(\mathrm{cm})$. The mean squares due to line $\mathrm{x}$ tester interaction were significant for plant height $(\mathrm{cm})$, pod bearing length $(\mathrm{cm})$, number of pods per plant and seed yield/plant $(\mathrm{g})$. Thereby it is sug-

Table 5. Comparison of best crosses on the basis of specific combining ability effects for different characters.

\begin{tabular}{|c|c|c|c|c|c|c|c|c|}
\hline \multirow[t]{2}{*}{ Characters } & \multirow[t]{2}{*}{ Crosses } & \multirow{2}{*}{$\begin{array}{l}\text { SCA } \\
\text { effects }\end{array}$} & \multicolumn{3}{|c|}{$\begin{array}{l}\text { GCA effects of Parents } \\
\text { and Status }\end{array}$} & \multirow{2}{*}{$\begin{array}{l}\text { Std. } \\
\text { het- } \\
\text { erosis }\end{array}$} & \multirow[t]{2}{*}{ per se } & \multirow{2}{*}{$\begin{array}{l}\text { Significant SCA } \\
\text { effects for other } \\
\text { traits }\end{array}$} \\
\hline & & & P1 & $\mathbf{P 2}$ & Status & & & \\
\hline \multirow{5}{*}{$\begin{array}{l}\text { Days to } 50 \% \\
\text { flowering } \\
\text { Days to } \\
\text { maturity } \\
\text { Plant height } \\
\text { (cm) } \\
\text { Number of } \\
\text { branches per } \\
\text { plant }\end{array}$} & ICPA-2078 X LRG-41 & -33.60 & -4.40 & -12.96 & L X L & 45.78 & 61.00 & NPP \\
\hline & ICPA-2043 X LRG-41 & -10.91 & 5.16 & -12.90 & HX L & 8.27 & 220.50 & DM, YLD \\
\hline & ICPA-2047 X ICP-6972 & 31.26 & 16.08 & -28.13 & HX L & 8.27 & 220.50 & DM, YLD \\
\hline & ICPA-2043 X TGT & & 441 & 11.03 & L X H & & & DM YID \\
\hline & ICPA-2047 X ICP-6972 & 3.99 & -0.27 & -3.83 & L X L & -23.38 & 13.66 & DM, PHT, YLD \\
\hline \multirow{3}{*}{$\begin{array}{l}\text { Pod bearing } \\
\text { length }(\mathrm{cm})\end{array}$} & ICPA-2043 X GRG-2OO9 & 17.83 & 1.51 & -5.10 & H X L & 21.88 & 78.00 & YLD, \\
\hline & ICPA-2078 X ICPL-89004 & 17.66 & -12.22 & 1.54 & L X H & 11.20 & 71.17 & NB, YLD \\
\hline & ICPA-2047 X LAXMI & 15.29 & 10.71 & 2.26 & H X H & 29.95 & 83.17 & DM, NB, YLD \\
\hline \multirow{3}{*}{$\begin{array}{l}\text { No. of pods/ } \\
\text { plant }\end{array}$} & ICPA-2043 X LRG-41 & 75.13 & 18.25 & -34.60 & H X L & 55.56 & 240.00 & YLD, \\
\hline & ICPA-2078 X MARUTI & 57.85 & -7.46 & -15.82 & $\mathrm{~L} X \mathrm{~L}$ & 6.96 & 165.17 & YLD, \\
\hline & ICPA-2078 X GRG-2OO9 & 57.41 & -7.46 & -20.54 & $\mathrm{~L} X \mathrm{~L}$ & -11.43 & 160.00 & NB, YLD \\
\hline \multirow{3}{*}{$\begin{array}{l}\text { Seed yield/ } \\
\text { plant }\end{array}$} & ICPA-2043 X GRG-2009-2 & 59.31 & 10.45 & 20.11 & H X H & 95.26 & 133.75 & - \\
\hline & ICPA-2047 X GRG-2OO9 & 21.52 & 1.11 & 18.60 & L X H & 24.23 & 85.10 & DM, PHT, NB \\
\hline & ICPA-2078 X LAXMI & 19.86 & -11.56 & 15.79 & $\mathrm{LX} \mathrm{H}$ & -17.72 & 56.36 & NB \\
\hline
\end{tabular}

$\mathrm{DFF}=$ Days to $50 \%$ flowering, $\mathrm{DM}=$ Days to maturity, $\mathrm{PHT}=$ Plant height $(\mathrm{cm}), \mathrm{NB}=$ No. of branches, $\mathrm{SB}=\mathrm{No}$. of Secondary branches, $\mathrm{PBL}=$ Pod bearing length $(\mathrm{cm}), \mathrm{NPPP}=$ No. of pods/ plant, YPP $=$ Seed yield/ plant. 
Table 6. Top three crosses based on per se for 7 yield and yield attributing characters in pigeonpea

\begin{tabular}{|c|c|c|c|c|c|c|}
\hline S. N. & Characters & Crosses & $\begin{array}{l}\text { Per se per- } \\
\text { formance }\end{array}$ & $\begin{array}{c}\text { Sca } \\
\text { effects }\end{array}$ & $\begin{array}{c}\text { GCA status of } \\
\text { parents } \\
\end{array}$ & Std. heterosis \\
\hline \multirow[t]{3}{*}{1} & Days to $50 \%$ & ICPA-2078 X LRG-41 & 90.00 & -33.60 & L X L & -14.78 \\
\hline & flowering & ICPA-2043 X ICP-6972 & 98.00 & -1.58 & L X L & -12.89 \\
\hline & & ICPA-2078 X ICP-6972 & 99.00 & 3.07 & L X L & -12.00 \\
\hline \multirow[t]{3}{*}{2} & Days to matur- & ICPA-2078 X LRG-41 & 140.00 & 1.56 & L X H & 1.90 \\
\hline & ity & ICPA-2043 X ICP-6972 & 143.00 & -1.08 & L X L & -9.21 \\
\hline & & ICPA-2078 X ICP-6972 & 144.00 & 1.89 & L X L & -8.57 \\
\hline \multirow[t]{3}{*}{3} & Plant height & ICPA-2047 X LRG-41 & 237.33 & 14.43 & H X H & 16.53 \\
\hline & & ICPA-2047 X GRG-2OO9 & 235.33 & 12.98 & H X H & 15.55 \\
\hline & & ICPA-2047 X TGT-501 & 232.67 & 4.26 & H X H & 14.24 \\
\hline \multirow[t]{3}{*}{4} & Branches & ICPA-2043 X GRG-295 & 23.84 & 3.93 & H X H & 33.64 \\
\hline & & ICPA-2043 X TS-3 & 20.16 & 2.09 & H X L & 13.06 \\
\hline & & ICPA-2043 X TGT-501 & 19.66 & 2.37 & H X L & 10.26 \\
\hline \multirow[t]{3}{*}{5} & bearing & ICPA-2047 X MARUTI & 93.83 & 8.29 & H X H & 46.61 \\
\hline & length & ICPA-2047 X WRP-1 & 93.67 & 1.51 & H X H & 46.35 \\
\hline & & ICPA-2047 X LAXMI & 83.17 & 15.29 & H X H & 29.95 \\
\hline \multirow[t]{3}{*}{6} & Number of pods & ICPA-2043 X LRG-41 & 240.00 & 75.13 & $\mathrm{H} \mathrm{X} \mathrm{H}$ & 55.56 \\
\hline & per plant & ICPA-2043 X JKM-197 & 206.17 & 12.97 & H X H & 35.06 \\
\hline & & ICPA-2043 X ICPL-288 & 197.83 & 18.36 & H X H & 28.05 \\
\hline \multirow[t]{3}{*}{7} & Seed yield per & ICPA-2043 X GRG-2009-2 & 133.75 & 59.31 & H X H & 95.26 \\
\hline & plant $(g)$ & ICPA-2047 X GRG-2009 & 85.10 & 21.52 & $\mathrm{HX} \mathrm{H}$ & 24.23 \\
\hline & & ICPA-2043 X ICPL-288 & 79.79 & 15.74 & $\mathrm{H} \mathrm{X} \mathrm{H}$ & 16.48 \\
\hline
\end{tabular}

gested that the variation in hybrids in respect of seed yield may be strongly influenced by the lines. The mean squares due to lines were larger in magnitude for most of the important yield attributes than testers indicating greater diversity amongst the lines as compared to testers (Table 2).

The analysis of variance for combining ability showed that the mean squares due to general and specific combining ability effects indicated involvement of both additive and nonadditive gene action. The mean sum of squares due to lines $\times$ testers and their interactions were highly significant for seed yield and it's component characters further indicated the importance of sca variance, and consequently the non-additive genetic variation in the inheritance of these characters. The trend recorded was in agreement with the findings of Khorgade et al. (2000), Sunil Kumar et al. (2003) and Sekhar et al. (2004). Vaghela et al.(2009), Sameer Kumar et al. (2009) Bharate et al. (2011) for seed yield/plant and other important yield attributes viz., pod bearing length, number of pods per plant and 100 seed weight. Preponderance of non-additive genetic variance has been suggested.

Per cent contribution of line, testers and line $\mathrm{x}$ testers to the crosses (Table 3 ) revealed that a greater contribution of testers was observed for days to $50 \%$ flowering, days to maturity, plant height, number of pods per plant and seed yield/plant. Whereas, the contribution of lines to the performance of crosses was found significant for pod bearing length and number of branches per plant indicating the significant contribution of paternal parents. The contribution of lines and testers were found equally important for the development of the yield and its attributing characters. This showed that average general combiner could results in high heterotic performance and could be effectively used in heterosis breeding programmes. Similar results were also reported by Pandey and Singh (2002) and Yamanura et al. (2014).

The nature and magnitude of combining ability effects help in identifying superior parents and their utilization in breeding programme. Character-wise estimation of gca effects of lines and testers is presented in table 4. The gca effects of parents revealed that ICPA2043, GRG-2009, GRG-2009-2, LAXMI, LRG-41 and JKM-197 were good general combiners for seed yield and it's direct components (Table 4). The line ICPA2078 and testers ICP-6972 and ICPL-89004 were good general combiners for days to $50 \%$ flowering and days to maturity, line ICPA-2047 and testers JKM-197 and TS-3 for plant height, line ICPA-2043 and testers GRG-295 and LAXMI for number of branches/plant, line ICPA-2047 and testers WRP-1 and MARUTI for Pod bearing length, line ICPA-2043 and testers JKM197, ICPL-288 and LRG-41 for number of pods/plant. The top three crosses exhibiting high specific combing ability effects along with their Per se performance, standard heterosis and gca status of the parents indicated that the cross combinations ICPA-2043 X GRG2009-2, ICPA-2047 X GRG-2OO9 and ICPA-2078 X LAXMI were good specific combiners for seed yield per plant. These parental combinations are being used for exploitation of hybrid vigour. The cross combination ICPA-2078 X LRG-41 and ICPA-2043 X LRG41 were good specific combiners for days to $50 \%$ flowering and maturity as they were showing highly significant negative $s c a$ effect this is very much suitable in dry land (rainfed) condition because it has advantage of escaping terminal moisture stress. The crosses ICPA-2047 X ICP-6972 and ICPA-2043 X TGT501for plant height, ICPA-2047 X ICP-6972 for number of branches, ICPA-2043 X GRG-2009, ICPA- 
Table 7. Per se performce of hybrids and parents in respect of 7 characters in Pigeopea.

\begin{tabular}{|c|c|c|c|c|c|c|c|c|}
\hline S.N. & Entries & $\begin{array}{c}\text { Days to } \\
50 \% \\
\text { flowering } \\
\end{array}$ & $\begin{array}{c}\text { Days to } \\
\text { maturity }\end{array}$ & $\begin{array}{c}\text { Plant } \\
\text { height } \\
(\mathrm{cm}) \\
\end{array}$ & $\begin{array}{l}\text { Number of } \\
\text { branches } \\
\text { per plant }\end{array}$ & $\begin{array}{c}\text { Pod bear- } \\
\text { ing length } \\
(\mathrm{cm})\end{array}$ & $\begin{array}{l}\text { No. of } \\
\text { pods/ } \\
\text { plant } \\
\end{array}$ & $\begin{array}{c}\text { Seed } \\
\text { yield/ } \\
\text { plant } \\
\end{array}$ \\
\hline 1 & ICPA-2043 X ICPL-288 & 110 & 155 & 200.33 & 18.17 & 73.50 & $198^{* *}$ & $79.79 * *$ \\
\hline 2 & ICPA-2043 X ICPL-89004 & 103 & $148^{*}$ & 179.50 & 12.84 & 56.50 & 153 & 38.09 \\
\hline 3 & ICPA-2043 X ICP-6974 & 111 & 156 & 198.67 & 16.67 & 73.33 & $190 *$ & 32.58 \\
\hline 4 & ICPA-2043 X ICP-7387 & 115 & 160 & 202.00 & 16.17 & 55.83 & 98 & 42.81 \\
\hline 5 & ICPA-2043 X ICP-6972 & 98 & $143 * *$ & 152.50 & 8.00 & 56.00 & 82 & 16.41 \\
\hline 6 & ICPA-2043 X LRG-41 & 105 & 150 & 196.00 & 18.00 & 68.17 & $240 * *$ & $72.52 * *$ \\
\hline 7 & ICPA-2043 X MARUTI & 113 & 161 & 202.00 & 14.83 & 69.50 & 96 & 41.15 \\
\hline 8 & ICPA-2043 X WRP-1 & 116 & 162 & 195.00 & 12.83 & 77.50 & 87 & 31.07 \\
\hline 9 & ICPA-2043 X GRG-2009 & 111 & 156 & 187.33 & 17.51 & 78.00 & 91 & $62.44 * *$ \\
\hline 10 & ICPA-2043 X JKM-197 & 127 & 175 & 205.67 & 16.83 & 65.83 & $206 * *$ & $63.36 * *$ \\
\hline 11 & ICPA-2043 X GRG-295 & 112 & 157 & 202.67 & $23.84 * *$ & 54.67 & 184 & $74.98 * *$ \\
\hline 12 & ICPA-2043 X TGT-501 & 111 & 156 & 208.33 & 19.66 & 62.17 & 155 & 50.46 \\
\hline 13 & ICPA-2043 X PG-12 & 104 & 149 & 191.50 & 14.83 & 59.67 & 123 & 52.31 \\
\hline 14 & ICPA-2043 X GRG-2009-2 & 111 & 156 & 217.33 & 16.00 & 64.83 & 144 & $133.75 * *$ \\
\hline 15 & ICPA-2043 X GRG-2009-3 & 111 & 156 & 195.50 & 15.66 & 64.67 & 148 & 41.77 \\
\hline 16 & ICPA-2043 X LAXMI & 122 & 167 & 217.33 & 16.00 & 64.83 & 144 & 32.33 \\
\hline 17 & ICPA-2043 X TS-3 & 114 & 159 & 200.00 & $20.16^{*}$ & 71.67 & $190 *$ & 57.84 \\
\hline 18 & ICPA-2078 X ICPL-288 & 103 & 148 & 174.33 & 9.16 & 62.67 & 115 & 23.15 \\
\hline 19 & ICPA-2078 X ICPL-89004 & 107 & 152 & 190.17 & 11.00 & 71.17 & 126 & 37.58 \\
\hline 20 & ICPA-2078 X ICP-6974 & 106 & 151 & 159.50 & 13.00 & 40.00 & 127 & 29.76 \\
\hline 21 & ICPA-2078 X ICP-7387 & 111 & 156 & 174.00 & 11.01 & 53.33 & 102 & 16.22 \\
\hline 22 & ICPA-2078 X ICP-6972 & 99 & $144^{*}$ & 146.50 & 8.17 & 48.83 & 102 & 18.15 \\
\hline 23 & ICPA-2078 X LRG-41 & 90 & $140 * *$ & 187.17 & 13.33 & 48.83 & 103 & 42.73 \\
\hline 24 & ICPA-2078 X MARUTI & 115 & 161 & 205.00 & 12.66 & 61.17 & 165 & 28.52 \\
\hline 25 & ICPA-2078 X WRP-1 & 113 & 156 & 210.33 & 9.84 & 73.17 & 120 & 29.10 \\
\hline 26 & ICPA-2078 X GRG-2009 & 112 & 157 & 196.17 & 11.51 & 21.50 & 160 & 39.89 \\
\hline 27 & ICPA-2078 X JKM-197 & 127 & 169 & 217.33 & 16.00 & 64.83 & 144 & 25.48 \\
\hline 28 & ICPA-2078 X GRG-295 & 113 & 158 & 195.83 & 14.16 & 61.33 & 122 & 30.46 \\
\hline 29 & ICPA-2078 X TGT-501 & 112 & 157 & 196.00 & 10.33 & 25.00 & 121 & 27.78 \\
\hline 30 & ICPA-2078 X PG-12 & 103 & $148^{*}$ & 155.00 & 8.67 & 47.17 & 103 & 23.19 \\
\hline 31 & ICPA-2078 X GRG-2009-2 & 112 & 152 & 192.83 & 12.34 & 64.67 & 187 & 35.42 \\
\hline 32 & ICPA-2078 X GRG-2009-3 & 113 & $148^{*}$ & 197.17 & 13.34 & 58.50 & 121 & 28.10 \\
\hline 33 & ICPA-2078 X LAXMI & 112 & 157 & 198.83 & 10.16 & 23.50 & 111 & 56.36 \\
\hline 34 & ICPA-2078 X TS-3 & 112 & 157 & 223.00 & 10.33 & 57.67 & 65 & 57.59 \\
\hline 35 & ICPA-2047 X ICPL-288 & 111 & 156 & 196.67 & 13.33 & 71.67 & 171 & 57.85 \\
\hline 36 & ICPA-2047 X ICPL-89004 & 108 & 153 & 209.17 & 12.84 & 69.50 & 143 & 50.67 \\
\hline 37 & ICPA-2047 X ICP-6974 & 122 & 167 & 216.00 & 13.50 & 70.83 & 119 & 35.25 \\
\hline 38 & ICPA-2047 X ICP-7387 & 120 & 165 & 224.50 & 17.67 & 75.50 & 146 & 46.98 \\
\hline 39 & ICPA-2047 X ICP-6972 & 104 & 149 & 220.50 & 13.66 & 58.50 & 104 & 27.09 \\
\hline 40 & ICPA-2047 X LRG-41 & 131 & 176 & $237.33 *$ & 12.83 & 78.00 & 97 & 43.24 \\
\hline 41 & ICPA-2047 X MARUTI & 112 & 159 & 227.00 & 13.50 & $93.83 * *$ & 83 & 35.11 \\
\hline 42 & ICPA-2047 X WRP-1 & 121 & 166 & $232.50 *$ & 12.84 & $93.67 * *$ & 133 & 30.68 \\
\hline 43 & ICPA-2047 X GRG-2009 & 123 & 168 & $235.33 *$ & 14.33 & 76.50 & 79 & $85.10^{* *}$ \\
\hline 44 & ICPA-2047 X JKM-197 & 128 & 169 & 227.00 & 13.17 & 74.17 & 174 & $56.33 * *$ \\
\hline 45 & ICPA-2047 X GRG-295 & 121 & 166 & 217.33 & 14.00 & 65.50 & 113 & 36.39 \\
\hline 46 & ICPA-2047 X TGT-501 & 112 & 157 & $232.67 *$ & 14.16 & 77.33 & 138 & 42.27 \\
\hline 47 & ICPA-2047 X PG-12 & 112 & 157 & 161.50 & 12.22 & 76.50 & 116 & 36.63 \\
\hline 48 & ICPA-2047 X GRG-2009-2 & 112 & 159 & 199.33 & 12.50 & 61.50 & 84 & 22.79 \\
\hline 49 & ICPA-2047 X GRG-2009-3 & 116 & 164 & 219.17 & 9.50 & 77.00 & 101 & 39.35 \\
\hline 50 & ICPA-2047 X LAXMI & 130 & 175 & 223.50 & 13.67 & $83.17 *$ & 66 & $55.48^{*}$ \\
\hline 51 & ICPA-2047 X TS-3 & 111 & 156 & 215.83 & 16.00 & 70.00 & 171 & $63.59 * *$ \\
\hline 52 & ICPA-2043 & 99 & $137 * *$ & 173.50 & 18.67 & 30.84 & 96 & 23.92 \\
\hline 53 & ICPA2078 & 92 & $135 * *$ & 117.17 & 9.01 & 11.92 & 56 & 12.25 \\
\hline 54 & ICPA-2047 & 111 & 167 & 213.83 & $20.67 *$ & 38.83 & 116 & 26.34 \\
\hline 55 & ICPL-288 & 122 & 167 & 175.00 & 14.16 & 63.33 & 162 & $70.49 * *$ \\
\hline 56 & ICPL-89004 & 112 & 157 & 164.50 & 11.34 & 55.50 & 135 & 22.91 \\
\hline 57 & ICP-6974 & 113 & 158 & 160.50 & 13.34 & 47.67 & 149 & 41.50 \\
\hline 58 & ICP-7387 & 126 & 159 & 166.83 & 13.84 & 42.17 & 102 & 24.34 \\
\hline 59 & ICP-6972 & 105 & 150 & 137.33 & 8.66 & 41.17 & 107 & 15.00 \\
\hline
\end{tabular}


Contd

\begin{tabular}{llccccccc}
\hline 60 & LRG-41 & 122 & 167 & 198.83 & 18.66 & 44.17 & $218^{* *}$ & 44.02 \\
61 & MARUTI & 113 & 150 & 203.67 & 17.84 & 64.00 & 155 & 41.23 \\
62 & WRP-1 & 114 & 153 & 197.67 & 10.67 & 74.83 & 148 & 38.92 \\
63 & GRG-2009 & 123 & 168 & 214.17 & 18.84 & 72.50 & 167 & 51.09 \\
64 & JKM-197 & 123 & 168 & 205.33 & 19.50 & 66.67 & 184 & $54.75^{*}$ \\
65 & GRG-295 & 121 & 166 & 193.83 & $20.50^{*}$ & 53.33 & 137 & $57.27^{* *}$ \\
66 & TGT-501 & 109 & 154 & 200.83 & 14.83 & 69.17 & 208 & $54.72^{*}$ \\
67 & PG-12 & 105 & 150 & 166.00 & 14.50 & 61.50 & 168 & $56.05^{*}$ \\
68 & GRG-2009-2 & 107 & 148 & 207.00 & 16.00 & 68.17 & $206^{* *}$ & $77.22^{* *}$ \\
69 & GRG-2009-3 & 108 & 151 & 210.67 & 14.50 & 66.67 & 145 & 40.44 \\
70 & TS-3 & 113 & 158 & 201.83 & $19.66^{*}$ & 57.67 & $195^{*}$ & $76.06^{* *}$ \\
71 & LAXMI & 122 & 167 & 205.83 & 13.00 & 66.33 & 140 & 51.66 \\
Geeral mean & 112 & 157 & 196.91 & 14.24 & 61.54 & 136 & 43.92 \\
CD $95 \%$ & 18.22 & 10.13 & 34.63 & 5.35 & 18.73 & 46.69 & 9.79 \\
CD $91 \%$ & 24.21 & 13.46 & 46.01 & 7.11 & 24.89 & 62.02 & 13.01 \\
\hline
\end{tabular}

2078 X ICPL-89004 and ICPA-2047 X LAXMI for pod bearing length, ICPA-2043 X LRG-41, ICPA2078 X MARUTI and ICPA-2078 X GRG-2OO9 for number of pods per plant were found to be useful. The estimates of $s c a$ effects revealed that 11 experimental hybrids had significant, desirable and positive sca effects for seed yield/plant. Among these, three best crosses were selected on the basis of per se performance for ascertaining their association with sca effects of seed yield per plant and its attributes (Table 5).

Out of three crosses showing high mean and significant positive $s c a$ effects for seed yield along with their per se performance as well as gca effects of parents and their significant response to other characters are presented in table 6 . The crosses ICPA-2043 X GRG2009-2, ICPA-2047 X GRG-2009 and ICPA-2043 X ICPL-288 showing high mean and significant positive $s c a$ effects involved high $\times$ high $g c a$ effects of parents. These results were also in conformity with those of Baskaran and Muthiah (2007), Meshram et al. (2013) and Chethana et al. (2013). Better performance of hybrids involving high $\mathrm{x}$ low or low $\mathrm{x}$ low general combiners indicated dominance $\mathrm{x}$ dominance (epitasis) type of gene action. The crosses showing high sca effects involving one good general combiner indicated additive $\mathrm{x}$ dominance type gene interaction which exhibit the high heterotic performance for yield and yield related traits.

The mean per se performance is an important parameter to be considered in selection of promising genotypes as they directly influence on other parameters like GCA, SCA and also on magnitude of heterosis in crosses. The mean per se performance of parents and crosses obtained in the present study were given in the table 7. Seed yield per plant is the effect of the component characters related to it. The potentiality of line to be used as a parent in hybridization, or in a cross to be used as a commercial hybrid may be judged by comparing the per se performance of the parents, the $F_{1}$ value and the combining ability effects.

The results suggested that the crosses having high mean performance, positive sca effects for seed yield and their significant response to other related traits had necessarily involved both or at least one parent as good combiner which could be commercially exploited for heterosis by taking advantage of high degree of natural out crossing in pigeonpea.

\section{Conclusion}

From the present study, the genotypes viz., ICPA2043, GRG-2009, GRG-2009-2, LAXMI, LRG-41 and JKM-197 were identified as good general combiners and ICPA-2043 X GRG-2009-2, ICPA-2047 X GRG2009 and ICPA-2043 X ICPL-288 as promising crosses having high mean and significant positive $s c a$ effects involving high $\times$ high $g c a$ effects of parents. These parental combinations may be used in breeding program for exploitation of hybrid vigour.

\section{ACKNOWLEDGEMENTS}

Sincere thanks to National Food Security Mission, New Delhi and International Crop Research Institute for Semi-arid and Tropics, Hyderabad for having provided seed materials and financial support under the project entitled "Taking pigeonpea projects to the door steps of the farmers".

\section{REFERENCES}

Bajpai, G.C., Jogendra Singh, Tewari, S.K. (2003). Pigeonpea hybrids - A review. Agric. Reviews, 24(1) 1-15.

Baskaran, K. and Muthiah, A.R. (2007). Combining ability studies in pigeonpea. Legume Research. 30: 67-69.

Beekham, A.P. and Umaharan. (2010). Inheritance and combining ability studies of pod physical and biochemical quality traits in vegetable pigeonpea. Euphytica. 176:36-47.

Bharate, B.S., Wadikar, P.B. and Ghodke, M.K. (2011). Studies on combining ability for yield and its components in pigeonpea. Journal of Food Legumes. 24(2): 148-149.

Chethana, C.K., Dharmaraj, P.S., Lokesha, R., Girisha, G., Muniswamy, S, Yamanura, Niranjana kumar and Vinayaka, D.H.(2013). Genetic analysis for quantitative traits in pigeonpea (Cajanus Cajan L. Millsp.). Journal of Food Legumes. 25(1): 1-18.

Kempthorne, O. (1957). An Introduction to Genetic Statistics. John Wiley and Sons Inc., New York; Chapman and Hall, London. .545. 
Khorgade, P.W., Wankhade, R.R. and Wanjari, K.B. (2000). Combining ability analysis in pigeonpea using male sterile lines. Indian Journal Agricultural Research. 34: 112-116.

Meshram, M.P., Patil, A.N. and Abhilasha, K. (2013). Combining Ability Analysis in Medium Duration CGMS Based Hybrid Pigeonpea (Cajanus cajan (L.) Millsp.,). Journal of Food Legumes. 26 (3 \& 4): 29-33.

Pandey, N. and Singh, N.B. (2002). Hybrid vigour and combining ability in long duration pigeonpea [Cajanus $\mathrm{ca}^{-}$ jan (L.) Millsp.] hybrids involving male sterile lines. Indian J. Genet. and Pl. Breed. 62(3): 221-225.

Panse, V.G. and Sukhatme, P.V. (1961). Statistical Methods for Agricultural Workers, ICAR Publication, New Delhi. P. 359.

Kumar, S., C.V. Sreelaxmi, C.H. and Kishore Verma, P. (2009). Studies on Combining ability and heterosis in pigeonpea [Cajanus cajan (L.) Millsp.]. Legume Research. 32(2): 92-97.

Saxena, K.B. and Sharma, D. (1990). Pigeonpea Breeding. The pigeonpea (Eds. Nene, Y.I., Hall, S.D. and Sheila,V. K.,). 375-399.

Saxena, K.B., Kumar, R.V., Dalvi, V.A., Mallikarjuna, N., Gowda, C.L.L., Singh, B.B., Tikka, S.B.S., Wanjari, K.B., Pandet, L.B., Paralkar, L.M., Patel, M.K., Shiying, B. and Xuxiao, Z. (2005). Hybrid breeding in grain legumes -a success story of pigeonpea. Khairwal MC, Jain HK (eds) Proceedings of the international food legumes research conference. New Delhi, India.
Sekhar, M.R., Singh, S.P., Mehra, R.B. and Govil, J.N. (2004). Combining ability and heterosis in early maturing pigeonpea [Cajanus cajan (L.) Millsp.] hybrids. Indian Journal of Genetics and Plant Breeding. 64 (3): $212-216$

Shobha, D. and Balan, A. (2010). Combining Ability in CMS/GMS Based Pigeonpea (Cajanus cajan (L.) Millsp.,) Hybrids. Madras Agricultural Journal. 97 (13): $25-28$

Sing, I.P. (2015). Project Coordinator's Report- Kharif Pulses. All Indian Coordinated Resaerch Project on Pigeonpea, Indian Institute of Pulses Research, Kanpur, India. P. $18 \& 24$

Tiwari, S. (2010). Estimation of heterosis, combining ability and genetic components for yield and yield attributes in Pigeonpea (Cajanus cajan L.). MSc (Agri) Thesis submitted to UAS, Raichur

Kumar, S., Lohithaswa, H.C., Dharmaraj, P.S. (2003). Combining ability analysis for grain yield, protein content and other quantitative traits in pigeonpea. J. Maharashtra Agric. Univ. 28(2): 141-144.

Vaghela, K.O., Desai, R.T., Nizama, J.R., Patel, J.D. and Sharma, V. (2009). Combining ability analysis in pigeonpea. Legume Research. 32 (4): 274-277.

Yamanura, Lokesha, R., Dharmaraj, P.S., Muniswamy, S. and Diwan, J.R. (2014). Estimation of Heterosis, Combining ability and Gene action in Pigeonpea [Cajanus cajan (L.) Millsp.]. Elect. J. of Plant. Breed., 5(2): 173-178. 